\section{Männer an die Sonne!}

$M$ angelt es an Vitamin D, sinkt der Testosteronspiegel, während Sexualhormon-bindendes Globulin im gleichen Maße ansteigt. Dies ergab eine Studie, für die Mediziner aus Graz, Ulm und Heidelberg Serumproben von rund 2.300 Männern mit einem Durchschnittsalter von 62 Jahren untersucht haben. Da die Bildung von Vitamin D von der Sonnenlichtexposition der Haut abhängt, ist der Vitamin-D-Spiegel im Sommer höher als in der dunklen Jahreszeit. Die Testosteronspiegel bilden diese jahreszeitlichen Schwankungen ebenfalls ab: mit einem Maximum im August und einem Tiefstand im März.

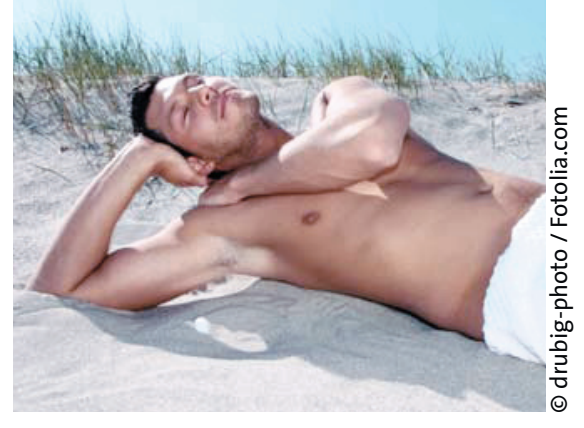

„Das könnte erklären, warum die Hormone im Frühling verrückt spielen“, mutmaßt Studienleiter Prof. Dr. Winfried März aus Heidelberg.

$d k a$

Wehr E et al. Clin Endocrinol 2009 Dec 29 [Epub ahead of print]

\title{
Autoinjektoren: Umsicht und Übung gefordert
}

W ie eine Datenerhebung in USamerikanischen Notfallzentren zeigt, nehmen unbeabsichtigte Adrenalin-Autoinjektionen zumindest jenseits des Atlantik deutlich zu. Insgesamt 15.190 Fälle wurden zwischen 1994 und 2007 dokumentiert, $60 \%$ davon allein zwischen 2003 und 2007. Betroffen waren erwartungsgemäß Personen mit Anaphylaxierisiko, aber auch deren Angehörige sowie medizinisches Personal. Im Mittel lag das Alter der Verunfallten bei nur 14 Jahren, was zeigt, dass vor allem
Kinder betroffen sind. Bei rund 30\% wurde eine Behandlung eingeleitet, bleibende Schäden hatte die Ungeschicklichkeiten beim Hantieren mit den Autoinjektoren aber offenbar in keinen Fall. Die Autoren der Studie fordern dennoch sicherer zu bedienende Pens und bitten alle Behandler dafür zu sorgen, dass ihre Patienten und deren Umfeld die Geräte auch sicher beherrschen.

Simons FE et al. J Allergy Clin Immuno 2010; 125: 419-23

\section{Probiotika als SLIT-Adjuvanzien}

W as die Kombination der klassischen sublingualen Immuntherapie (SLIT) mit probiotischen Bakterien bringt, wollten französische Forscher wissen: Im Tierversuch waren die Bakterien in der Lage, bei oraler Applikation durch den Kontakt mit der Schleimhaut die Antworten von dendritischen Zellen und T-Zellen zu beeinflussen. Für die Hyposensibilisierung kommen aber nur solche Probiotika in Frage, die sowohl TH1- wie auch regulatorische $\mathrm{T}$-Zellen stimulieren, reine TH1-Adjuvanzien scheinen die Wirksamkeit der oralen Allergentherapie nicht zu verbessern. So erhöhte im Mausmodell der Zusatz von Lactobacillus helveticus die Wirkung einer SLIT deutlich, während der lediglich TH1-modulierende Stamm L. casei keinen therapeutischen Zusatznutzen hatte. $\quad b k$

Overtvelt $L$ van et al. Vaccine 2010 Feb 20 [Epub ahead of print]

\section{Sechster Sinn für Fett}

isher ging man davon aus, dass der Mensch fünf Grundgeschmacksrichtungen unterscheiden kann: süß, salzig, bitter, sauer und umami. Umami bedeutet im Japanischen fleischig, herzhaft, wohlschmeckend. Proteinreiche Nahrung schmeckt „umami“. Forscher in Neuseeland haben jetzt einen sechsten Geschmackssinn entdeckt: fettig. Die Fähigkeit, Fett zu schmecken, ist nicht bei allen Menschen gleich ausgeprägt. Wer einen stark entwickelten Fettgeschmackssinn hat, konsumiert weniger Fett und neigt weniger zu Übergewicht als Personen mit einem unterentwickelten Fettgeschmackssinn.

Stewart JE. Br J Nutr 2010 Mar 3 [Epub ahead of print]

\section{Immuntherapie wirkt}

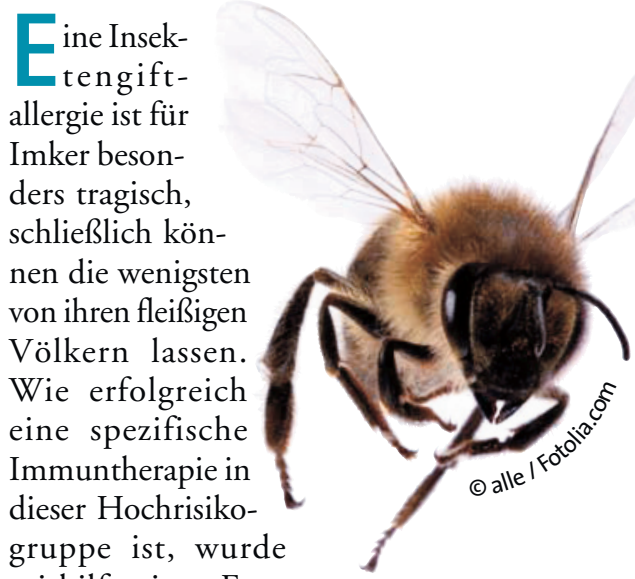
mithilfe einer Frage-

bogenaktion untersucht. Bei 43 Imkern, die sich einer InsektengiftHyposensibilisierung unterzogen hatten, war es in der Folge zu zahlreichen Bienenstichen - im Mittel 107 - gekommen, die aber bis auf eine Ausnahme alle ohne systemische Reaktion vertragen hatten. Diese Ergebnisse bestätigen die gute Wirksamkeit der Immuntherapie, die bei Bienengift um $85 \%$ liegt. Dennoch gilt auch hier die Empfehlung, den Anaphylaxie-Auslöser zu meiden - sprich die Imkerei aufzugeben.

Münstedt $\mathrm{K}$ et al. J Investig Allergol Clin Immunol 2010; 20: 58-62 